\title{
Effect of vitamin D on sleep quality in hemodialysis patients
}

\author{
Narges Sadat Zahed ${ }^{1}$, Maryam Namakchian $^{2^{*}}{ }^{\circledR}$ Zahra Davoudi $^{3^{\circledR}}$, Adineh Taherkhani $^{\left.{ }^{(}\right)}$ \\ ${ }^{1}$ Department of Adult Nephrology, School of Medicine, Loghman Hakim Hospital, Shahid Beheshti University of Medical Sciences, Tehran, Iran \\ ${ }^{2}$ Department of Internal Medicine, School of Medicine, Loghman Hakim Hospital, Shahid Beheshti University of Medical Sciences, Tehran, Iran \\ ${ }^{3}$ Department of Endocrinology, School of Medicine, Loghman Hakim Hospital, Shahid Beheshti University of Medical Sciences, Tehran, Iran
}

\begin{tabular}{l} 
A R T I C L E I N F O \\
\hline Article Type: \\
Original \\
\hline \\
Article History: \\
Received: 19 June 2020 \\
Accepted: 5 September 2020 \\
Published online: 8 October 2020
\end{tabular}

Keywords:

Sleep quality

Vitamin D

Hemodialysis

\begin{abstract}
A B S T R A C T
Introduction: Quality of sleep is among the factors that affect the improvement of life quality. The previous studies showed that $50 \%-80 \%$ of hemodialysis patients experience sleeping disturbances. Additionally, dialysis patients commonly experience vitamin D deficiency.

Objectives: We aimed to determine the impact of vitamin D deficiency therapy on sleep quality of hemodialysis patients.

Patients and Methods: Thirty hemodialysis patients with 25-hydroxy vitamin D deficiency and Pittsburgh Sleep Quality Index (PSQI) $\geq 5$ were enrolled in this clinical trial. Patients were treated with 50000-unit vitamin D per week for 12 weeks. After treatment, the PSQI score was recalculated for each patient.

Results: Nineteen out of 30 patients (63.3\%) were men and $11(36.7 \%)$ were women, with a mean age of $56.7 \pm 14.3$ years. The mean of vitamin D level was $18.61 \pm 6.32 \mathrm{ng} / \mathrm{mL}$ before treatment and $41.14 \pm 9.62 \mathrm{ng} / \mathrm{mL}$ after the treatment. The mean of PSQI score at the start of study was 9.97 and after treatment with vitamin D3, it was $9.47(P>0.05)$.

Conclusion: Treatment of vitamin D deficiency did not have any effect on the sleep quality according to the PSQI score in hemodialysis patients.

Trial Registration: This randomized controlled trial was registered in the Iranian Registry of Clinical Trials (identifier: IRCT20200223046593N1; https://en.irct.ir/trial/46126, ethical code; IR.SBMU.RETECH.REC.1397.1277).
\end{abstract}

Implication for health policy/practice/research/medical education:

In a study on 30 hemodialysis patients with vitamin D deficiency and poor sleep quality, we found that the treatment of vitamin D deficiency has no effect on sleep quality according to Pittsburgh Sleep Quality Index (PSQI).

Please cite this paper as: Zahed NS, Namakchian M, Davoudi Z, Taherkhani A. Effect of vitamin D on sleep quality in hemodialysis patients. J Renal Inj Prev. 2021; 10(4): e32. doi: 10.34172/jrip.2021.32.

\section{Introduction}

Sleep disorder is commonly experienced by hemodialysis (HD) patients. Previous studies showed that about $50 \%-80 \%$ of HD patients suffered from sleep disorders (dyssomnia) (1-3). These disorders consist of insomnia (problems in the start of sleeping and/or repeated waking in the nights) and feeling sleepy during the daytime, restless legs syndrome and/or periodical limb movements and sleep apnea $(1,4)$. Sleep quality significantly contributes to the improvement of life quality $(2,5,6)$. Sleep disorders are associated with impairment in immune functioning $(1,7)$, increased mortality and cardiovascular risk $(2,5,6)$. There is an association between sleep quality of HD patients and the following factors; female gender, older age, caffeine consumption, alcohol consumption, tobacco abuse, concurrent comorbidity, recombinant erythropoietin therapies, years receiving dialysis, dialysis shift, depression, physical performance, greater body mass index (BMI) values, dialysis efficiency, anemia, hypoalbuminemia, parathyroid hormone and serum creatinine (8-10). Deficiency of vitamin D is commonly experienced by patients who undergo dialysis $(6,11,12)$. Several studies reported the relationship of vitamin D deficiency and sleep disorders in non-dialysis $(13,14)$ and dialysis patients $(6,15)$. Also, according to several research works, vitamin D deficiency treatment improves sleep quality in non-dialysis patients $(16,17)$. 


\section{Objectives}

The present study aims at determining the effect of vitamin $\mathrm{D}$ deficiency treatment on the sleep quality of patients receiving $\mathrm{HD}$.

\section{Patients and Methods}

\section{Study population}

This clinical trial was conducted at HD center of Loghman Hakim hospital in Tehran, Iran from May 2018 to June 2019. The inclusion criteria were the following; being above 18 years, receiving dialysis for a minimum of three months, HD adequacy (Kt/V $>1 / 2)$, 25-hydroxy vitamin D $(25(\mathrm{OH}) \mathrm{D})<30 \mathrm{ng} / \mathrm{mL}$, and Pittsburgh Sleep Quality Index (PSQI) $\geq 5$. Psychiatric disorders (clinical diagnosis and or previous treatment), drug abuse, alcohol consumption, smoking, habits such as having coffee or tea prior to sleep and any hospitalization record during the previous four weeks were considered as the exclusion criteria.

First, the serum level of vitamin D was measured by enzyme-linked immunosorbent assay (ELISA) in the morning venous blood sample. Then, we completed the Pittsburg Sleep Quality questionnaire for patients with vitamin D deficiency. Out of $200 \mathrm{HD}$ patients, only 30 individuals met the inclusion criteria (Figure 1). We employed the PSQI to collect information through selfreports to determine the quality as well as the quantity of sleep during one month before assessment. PSQI consists of 19 individual items, which create seven components of subjective sleep quality, sleep latency, length of sleep, habitual sleep efficiency, sleep disturbance, taking sleep medicine and dysfunctions during the day, which provide one global score between 0-21. Scores of five or higher suggested a poorer sleep. This questionnaire has validity and reliability (18). Because of vitamin D deficiency complications in people receiving $\mathrm{HD}$ and its ethical considerations, all patients with vitamin D deficiency were treated. Confounding factors were controlled too. Treatment was performed using 50000 units of vitamin D 3/week over a 12-week interval. Measurement of the serum 25(OH)D levels as well as PSQI scores was carried out following the treatment. Patients were revisited and excluded if they met exclusion criteria after 12 weeks, although all patients remain in the trial.

\section{Statistical analysis}

Data were analyzed by SPSS 24, while data distribution was considered using paired $t$ test. Mean \pm SD or median

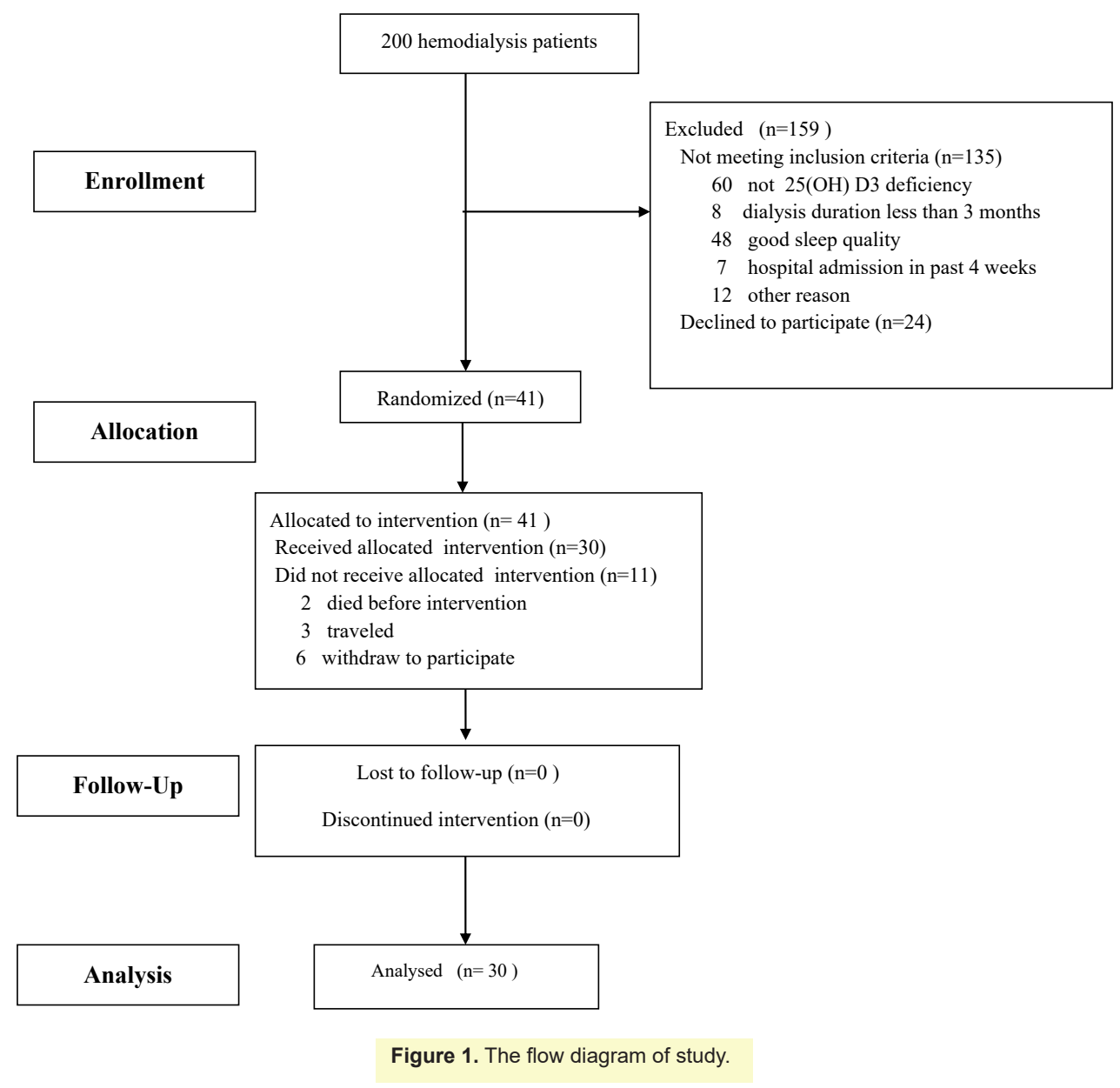


was used to show the study results regarding normal or non-normal distribution of continuous variables along with numbers or percentages for the nominal variables. $P$ value of $<0.05$ was set as the significance level.

Results

Table 1 presents baseline demographics. Research participants included 19 males and 11 females. The age distribution was between 32 and 85 years, with a mean age of $56.7 \pm 14.31$ years. Etiologic findings of ESRD indicated diabetes in $12(40 \%)$ and hypertension in $8(26.7 \%)$ patients. The mean of the duration of HD was $42.20 \pm$ 40.28 months. The participants' mean of vitamin D level was $18.61 \pm 6.32 \mathrm{ng} / \mathrm{mL}$ before treatment and $41.14 \pm 9.62$ $\mathrm{ng} / \mathrm{mL}$ after treatment. A mean PSQI score of 9.97 was obtained at week 0 while it was 9.47 (mean difference 0.005) after 12 weeks that did not show statistical significance $(P=0.473)$.

Habitual sleep efficiency significantly changed after treatment $(P=0.037)$, meaning that the number of hours slept/number hours spent in bed increased (Table 2).

Table 1. Demographic features of patients

\begin{tabular}{lc}
\hline Parameter & Value \\
\hline Age (years) & \\
Mean \pm SD & $56.70 \pm 14.31$ \\
Median & 56.50 \\
Range (Minimum-Maximum) & $53(32-85)$ \\
Gender & \\
Female & $11(36.7 \%)$ \\
Male & $19(63.3 \%)$ \\
Dialysis duration (months) & \\
Mean \pm SD & $42.2 \pm 40.28$ \\
Median & 30.5 \\
Scope (Minimum-Maximum) & $165(3-168)$ \\
Etiology of ESRD & \\
DM & $12(40.0 \%)$ \\
HTN & $8(26.7 \%)$ \\
Other causes & $10(33.3 \%)$ \\
\hline
\end{tabular}

Discussion

Based on our study, PSQI score did not change significantly after the treatment of vitamin $\mathrm{D}$ deficiency. Prior literature indicates the relationship of vitamin D deficiency with the quality of sleep in dialysis patients, but no study has been performed on the treatment of vitamin $\mathrm{D}$ deficiency in HD patients.

Hon et al and Yavuz et al approved that the levels of $25(\mathrm{OH}) \mathrm{D}$ fell down in dialysis patients having sleep disturbances, in comparison with those that do not have sleep disorders $(6,15)$. However, they did not confirm whether the treatment of vitamin D deficiency could affect the quality of sleep. Improvement of sleep quality with vitamin D supplement was reported in some studies. Gominak et al showed sleep improvement with $25(\mathrm{OH})$ $\mathrm{D}$ supplement in individuals who suffer neurologic problems and had evidence of abnormal sleep; however, only a limited range of $25(\mathrm{OH}) \mathrm{D}$ blood level of $60-80$ $\mathrm{ng} / \mathrm{mL}$ was maintained (16). This level of vitamin $\mathrm{D}$ is not recommended in dialysis patients, therefore we kept vitamin D level between $30-40 \mathrm{ng} / \mathrm{mL}$, according to other studies. In another study, which was conducted on patients with chronic pain, sleep latency, sleep duration and global PSQI score improved with 25(OH)D supplement (17).

Sahakyan reported insomnia improvement with a combination of vitamin D and melatonin in 60-65-yearold women with vitamin D deficiency (19).

The difference of our study is that most studies were carried out on non-HD people. The second reason may be other confounding factors not yet found. The small number of participants may also be the other reason for this difference.

Kidir et al examined the effect of vitamin D on sexual dysfunction in 37 dialysis patients (40.5\% HD patients and $59.5 \%$ peritoneal dialysis patients). They measured several parameters including sleep disorders and sleep score, and found that vitamin D treatment for 6 months did not affect sleep score (20).

Vitamin D deficiency has many complications in dialysis patients, hence all these patients must be treated.

Table 2. Mean and mean difference of components of PSQI before and after treatment of vitamin D deficiency

\begin{tabular}{|c|c|c|c|c|c|c|}
\hline \multirow{2}{*}{ Component of PSQI } & \multirow{2}{*}{$\begin{array}{l}\text { Mean before } \\
\text { intervention }\end{array}$} & \multirow{2}{*}{$\begin{array}{l}\text { Mean after } \\
\text { intervention }\end{array}$} & \multirow{2}{*}{$\begin{array}{c}\text { Mean } \\
\text { difference }\end{array}$} & \multicolumn{2}{|c|}{$95 \% \mathrm{Cl}$ of the difference } & \multirow{2}{*}{$P$ value } \\
\hline & & & & Lower & Upper & \\
\hline Subjective sleep quality & 1.40 & 1.23 & 0.167 & -0.160 & 0.493 & 0.305 \\
\hline Sleep latency & 1.67 & 1.80 & -0.133 & -0.611 & 0.344 & 0.573 \\
\hline Sleep duration & 1.87 & 1.73 & 0.133 & -0.173 & 0.439 & 0.380 \\
\hline Habitual sleep efficiency & 1.90 & 1.40 & 0.500 & 0.320 & 0.968 & $0.037^{a}$ \\
\hline Sleep disturbances & 1.37 & 1.20 & 0.167 & -0.075 & 0.409 & 0.169 \\
\hline Use of sleeping medication & 0.80 & 1.10 & -0.300 & -0.656 & 0.056 & 0.095 \\
\hline Daytime dysfunction & 0.93 & 0.97 & -0.033 & -0.498 & 0.432 & 0.884 \\
\hline Global PSQI Score & 9.97 & 9.47 & 0.500 & -0.907 & 1.907 & 0.473 \\
\hline
\end{tabular}

Abbreviation: $\mathrm{Cl}$, confidence interval; ${ }^{2}$ Statistically significant. 


\section{Conclusion}

Treatment of vitamin D deficiency does not affect the quality of sleep according to PSQI in HD patients. However, habitual sleep efficiency significantly changed after treatment.

\section{Limitations of the study}

The subjective nature of the PSQI could lead to limitations on its value in cases where some patients did not cooperate well. Moreover, another limitation was the small sample size of our study.

\section{Acknowledgments}

The authors would like to thank the Clinical Research Development Unit of Loghman Hakim hospital, Shahid Beheshti University of Medical Sciences, Tehran, Iran for their support, cooperation and assistance throughout the period of study. This study was presented as a poster at the World Congress of Nephrology 2020.

\section{Authors' contribution}

ZNS; preparation of research design and manuscript drifting. DZ and TA; manuscript reviewing. MN; data collection and statistical analysis. All authors read and signed the final paper.

\section{Conflicts of interest}

No conflicts of interest were declared by the researchers.

\section{Ethical issues}

The study was conducted based on the Declaration of Helsinki, and the patients filled out informed consent. Moreover, the Ethical Committee of Shahid Beheshti University of Medical Sciences declared the approval of the research (IR.SBMU.RETECH.REC.1397.1277). This work has been extracted from the internal medicine residential thesis of Maryam Namakchian in the School of Medicine, Shahid Beheshti University of Medical Sciences. Besides that, the study protocol was registered in the Iranian registry of clinical trials (identifier: IRCT20200223046593N1; https://en.irct.ir/trial/46126). Moreover, ethical considerations consisting of plagiarism, misconduct, data fabrication, falsification, double publications or submissions, and redundancies were thoroughly considered by the authors.

\section{Funding/Support}

Clinical Research Development Center of Loghman Hakim hospital, Shahid Beheshti University of Medical Sciences provided the necessary financial support for the present study (Grant number: 14843).

\section{References}

1. Chen WC, Lim PS, Wu WC, Chiu HC, Chen CH, Kuo $\mathrm{H}-\mathrm{Y}$, et al. Sleep behavior disorders in a large cohort of Chinese (Taiwanese) patients maintained by long-term hemodialysis. Am J Kidney Dis. 2006;48:277-84. doi: 10.1053/j.ajkd.2006.04.079

2. Maung SC, El Sara A, Chapman C, Cohen D, Cukor D. Sleep disorders and chronic kidney disease. World J Nephrol. 2016;5:224. doi: 10.5527/wjn.v5.i3.224

3. Forni Ogna V, Ogna A, Pruijm M, Bassi I, Zuercher E, Halabi G, et al. Prevalence and diagnostic approach to sleep apnea in hemodialysis patients: a population study. BioMed Res Int. 2015;2015:103686. doi: 10.1155/2015/103686.

4. Koch BC, Nagtegaal JE, Kerkhof GA, Ter Wee PM. Circadian sleep-wake rhythm disturbances in end-stage renal disease. Nat Rev Nephrol. 2009;5:407. doi: 10.1038/ nrneph.2009.88

5. Chen JY, Lin YC, Chen HH, Kao CC, Chang FC, Chen $\mathrm{TW}$, et al. The association between biochemical parameters and sleep related disorders in hemodialysis patients. Acta Nephrologica. 2016;30:79-86. doi: 10.6221/AN.2016032

6. Han B, Zhu FX, Shi C, Wu HL, Gu XH. Association between serum vitamin D levels and sleep disturbance in hemodialysis patients. Nutrients. 2017;9(2):139. doi: $10.3390 /$ nu 9020139

7. Irwin M. Effects of sleep and sleep loss on immunity and cytokines. Brain Behav Immun. 2002;16:503-12. doi: 10.1016/s0889-1591(02)00003-x

8. Danielle FMEH, Mahamat M, Francois KF, Marie-Patrice $\mathrm{H}$, Gloria A. Sleep quality on maintenance hemodialysis patients in Douala General Hospital in Cameroon. Open J Nephrol. 2017;7(3):61. doi:10.4236/ojneph.2017.73008

9. Elder SJ, Pisoni RL, Akizawa T, Fissell R, Andreucci VE, Fukuhara S, et al. Sleep quality predicts quality of life and mortality risk in haemodialysis patients: results from the Dialysis Outcomes and Practice Patterns Study (DOPPS). Nephrol Dial Transplant. 2008;23:998-1004. doi: 10.1093/ ndt/gfm630

10. Gusbeth-Tatomir P, Boisteanu D, Seica A, Buga C, Covic A. Sleep disorders: a systematic review of an emerging major clinical issue in renal patients. Int Urol Nephrol. 2007;39:1217-26. doi: 10.1007/s11255-007-9262-2

11. Jean G, Souberbielle JC, Chazot C. Vitamin D in chronic kidney disease and dialysis patients. Nutrients. 2017;9:328. doi: $10.3390 /$ nu 9040328

12. Bhan I, Dobens D, Tamez H, Deferio JJ, Li YC, Warren HS, et al. Nutritional vitamin D supplementation in dialysis: a randomized trial. Clin J Am Soc Nephrol. 2015;10:611-9. doi: 10.2215/CJN.06910714

13. Kim JH, Chang JH, Kim DY, Kang JW. Association between self-reported sleep duration and serum vitamin D level in elderly Korean adults. J Am Geriatr Soc. 2014;62:2327-32. doi: 10.1111/jgs.13148

14. Massa J, Stone KL, Wei EK, Harrison SL, Barrett-Connor E, Lane NE, et al. Vitamin D and actigraphic sleep outcomes in older community-dwelling men: the MrOS sleep study. Sleep. 2015;38:251-7. doi: 10.5665/sleep.4408

15. Yavuz D, Demirag MD, Yavuz R, Karagoz Ozen DS, Ramazanoglu Z. 25-Hydroxy vitamin D level is associated with sleep disturbances in patients with chronic kidney disease on hemodialysis: a cross-sectional study. Turk J Med Sci. 2020;50:298-303. doi: 10.3906/sag-1908-87

16. Gominak SC, Stumpf W. The world epidemic of sleep disorders is linked to vitamin D deficiency. Med Hypotheses. 
2012;79:132-5. doi: 10.1016/j.mehy.2012.03.031

17. Huang W, Shah S, Long Q, Crankshaw AK, Tangpricha V. Improvement of pain, sleep, and quality of life in chronic pain patients with vitamin D supplementation. Clin J Pain. 2013;29:341-7. doi: 10.1097/AJP.0b013e318255655d

18. Buysse DJ, Reynolds CF, Monk TH, Berman SR, Kupfer DJ. The Pittsburgh Sleep Quality Index: a new instrument for psychiatric practice and research. Psychiatry res. 1989;28:193-213. doi: 10.1016/0165-1781(89)90047-4

19. Sahakyan G. The role of Vitamin D in treatment of Chronic Insomnia with Melatonin (P5. 320). Neurology. 2018;90.

20. Kidir V, Altuntas A, Inal S, Akpinar A, Orhan H, Sezer MT. Sexual dysfunction in dialysis patients: does vitamin D deficiency have a role? Int J Clin Exp Med. 2015;8:22491.

Copyright (c) 2021 The Author(s); Published by Nickan Research Institute. This is an open-access article distributed under the terms of the Creative Commons Attribution License (http://creativecommons.org/licenses/by/4.0), which permits unrestricted use, distribution, and reproduction in any medium, provided the original work is properly cited. 